\title{
Novel Three-Dimensional Beam Tracking System for Stationary-Sample-Type Atomic Force Microscopy
}

\author{
Shao-Kang Hung, Student Member, IEEE, and Li-Chen Fu, Fellow, IEEE
}

\begin{abstract}
The stationary-sample (scanning-probe)-type atomic force microscope (AFM) has been demonstrated to have many advantages over its conventional counterpart: the scanningsample (stationary-probe)-type AFM. However, its major challenge is to measure the deflection of the probe while moving in three-dimensional (3-D) space. Utilizing a distinctively arranged correction lens in the optomechatronic integrated design, this paper proposes a novel laser beam tracking system to overcome the aforementioned challenge. An innovative method to minimize "false deflection" is devised. This system has been verified to achieve high scanning speed without sacrificing high tracking accuracy.
\end{abstract}

Index Terms-Atomic force microscope (AFM), nanotopography, optical position measurement, optomechatronics, scanning probe microscope (SPM).

\section{INTRODUCTION}

A TOMIC force microscope (AFM) [1] can produce threedimensional (3-D) images of a surface with nanometer resolution. Conventionally, the sample is carried to scan by a piezoelectric scanner, while the AFM probe is kept stationary (scanning-sample-type AFM). However, problems arise for large or heavy samples, such as semiconductor wafers, because the mass of the sample may reduce the bandwidth of the scanner. Temperature control (heating or cooling) of the sample may also affect the performance of the piezoelectric scanner. For samples (such as bio molecules) in a liquid cell, scanningsample-type AFM may also cause some problems.

The stationary-sample-type AFM, in which the probe is scanned while the sample is kept stationary, has been developed to solve the aforementioned problems. One way to achieve this is to let the whole optical detecting system move with the probe. However, the optical detecting set, including the laser diode (LD), the position-sensitive detector (PSD), alignment

Manuscript received June 15, 2004; revised April 27, 2006. This work was supported in part by the National Science Council, R.O.C., under Grant NSC94-2752-E-002-007-PAE.

S.-K. Hung is with the Department of Electrical Engineering, National Taiwan University, Taipei 106, Taiwan, R.O.C., and also with the Nanoscience Laboratory, Institute of Physics, Academia Sinica, Taipei 106, Taiwan, R.O.C. (e-mail: skhung@ntu.edu.tw).

L.-C. Fu is with the Department of Electrical Engineering, National Taiwan University, Taipei, Taiwan 106, R.O.C., and also with the Department of Computer Science and Information Engineering, National Taiwan University, Taipei, Taiwan 106, R.O.C., and the Tjing Ling Industrial Research Institute, National Taiwan University, Taipei, Taiwan 106, R.O.C. (e-mail: lichen@ntu.edu.tw).

Digital Object Identifier 10.1109/TIM.2006.881571 mechanisms, and the frame structure for maintaining the optical configuration, is often too massive to be driven by the scanner. Some methods have been proposed to reduce the total mass of the moving parts. The tracking lens method [2] is a typical solution to deal with this problem. In such design, however, the tracking error can limit its resolution. For an ideal optical tracking, when the laser beam emitted from the stationary LD perfectly tracks the moving probe, the signal picked up by the PSD depends only on the deflection of the probe and not on the scanning motion. If the PSD signal changes during scanning while the deflection of the probe is null, such situation is called "false deflection" or optical tracking error. The experimental method to measure false deflection is to operate far away from the sample or even without any sample. During such void scanning, the measured deflection is false deflection since there is no force to deflect the probe.

To reduce false deflection, a one-dimensional beam tracking method that makes the PSD move synchronously with the probe was introduced [3]. Hansma and Drake proposed another method that sets up a convex lens before the PSD to reduce false deflection [4]. In their system, however, the tracking function applies to the horizontal direction but not to the vertical direction. On the other hand, the tracking function was designed only for tube-type scanners and not for flexure-type scanners, which have better in-plane motion accuracy. A 3-D beam tracking system by using tracking mirrors was later proposed by Nakano [5]. In such system, the working distance from the LD to the reflection point of the probe changes during scanning. This means that the intensity signal of the beam varies if some portion of the beam falls off the probe due to the defocus of the laser spot on the probe. If this occurs, the shape of the reflected beam will be warped on the PSD, and the PSD position signal will also be adversely affected. A special type of twistprobe was used. It has a large mirror portion and can reduce the falloff, but its installation will readily limit one dimension of the image size. Another disadvantage of the twist-probe is that its distance to the PSD may change during scanning so that the constant relationship between probe deformation and the PSD signal cannot be guaranteed.

The objective of this paper is to develop a new 3-D laser beam tracking AFM system applicable to flexure-type scanners and the case of widely used bending mode probes. The aim is to limit both vertical and horizontal tracking errors to $<0.3 \mathrm{~nm}$ over a $4-\mu \mathrm{m}$ scanning distance and to $<12 \mathrm{~nm}$ over a $100-\mu \mathrm{m}$ scanning distance. 


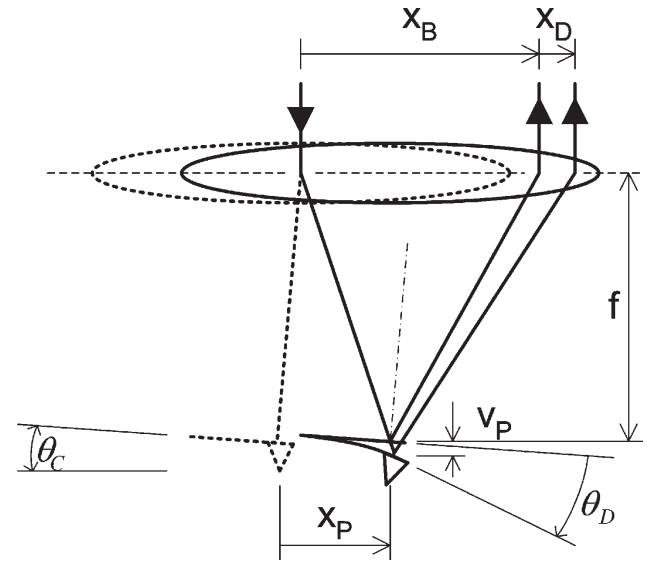

Fig. 1. Optical-lever technique used to detect the deflection of the cantilever. The reflected laser beam shifts from the incident laser beam by vector $x_{B}$ when there is no deflection. When the deflection occurs, an additional laser beam translation $x_{D}$ will be detected by the PSD. In the case of AFM, it can be shown that $x_{D}$ is much more contributed from the inclination angle $\theta_{D}$ rather than the displacement $v_{P}$.

\section{PRoblem Definition}

In most AFM systems, the optical lever technique is used to measure the probe's deflection, which is caused by the interaction force between the probe and the sample. Fig. 1 illustrates the optical configuration around the probe. It can be seen that a nonzero tilt angle $\theta_{C}$ is needed to make sure that the tip of the probe is the lowest point to touch the sample. Generally speaking, the range $10^{\circ}-15^{\circ}$ is reasonable; hence, $12^{\circ}$ is chosen in the present design. Moreover, $f$ is the focal length of the objective lens, which is $9 \mathrm{~mm}$. When the deflection occurs, the PSD measures the laser beam translation and then generates a voltage signal, which only depends on the probe's deflection. This signal is fed into a controller so as to form a feedback loop that forces the sample up and down in order to keep the tip-sample gap constant. Then, we can record the voltage applied to the $z$ scanner as the sample's profile. This voltage signal fails to represent the $z$ scanner's trajectory perfectly and may give only semiquantitative data because of the piezoelectric nonlinearities, such as hysteresis and creep. In order to measure the true trajectory, high-resolution sensors should be adopted. Strain gauges, parallel capacitor pairs, and optical fiber interferometers [6], [7] are commonly used. The other cost-effective method used in this paper is to calibrate the scanner [8]. By scanning a known sample (calibration gratings TGQ1, NT-MDT Company) and confirming that the obtained structure accords with what its producer claims, we can then guarantee that the acquired images are correct.

Butt and Jaschke [9] have shown that what we measured is a virtual deflection $v_{P}^{*}$, which is caused by the inclination angle $\theta_{D}$, rather than the actual deflection $v_{P}$. In the case of a rectangular cantilever with a free end, the virtual deflection should be corrected with the real one using the following relation:

$$
\sqrt{\left(v_{P}^{*}\right)^{2}}=\sqrt{\frac{4}{3} v_{P}^{2}}
$$

The linear relation between the deflection $v_{P}$ and the measurement $\Delta x_{B}$ can be verified in the force curve experiment [10]. Equation (2), which is shown below, expresses this relation. Lévy and Maaloum [10] have shown that coefficient $K$ can be evaluated via the slope of the force curve

$$
v_{P}=K \cdot \Delta x_{B} .
$$

Due to the variation in every silicon-fabricated probe, this coefficient $K$ should be calibrated whenever a new probe is loaded to replace the worn-out one. Equation (2) allows us to deduce the deflection $v_{P}$ from the measurement $\Delta x_{B}$ by using PSD. The resolution of the PSD (SPOT-4D, UDT Sensors Inc.) used for this paper is $0.1 \mu \mathrm{m}$. The coefficient $K$ of the probe (CONT-10, Nanosensors Company) used in the succeeding experiments is measured as 0.0011 , and its spring constant is $0.2 \mathrm{~N} / \mathrm{m}$. According to (2), the resolvable probe deflection and the interaction force are $0.11 \mathrm{~nm}$ and $22 \mathrm{pN}$, respectively, which is the reason AFM has the capability to resolve nanostructures.

In the conventional scanning-sample-type AFM, the 3-D motion of the sample is carried out by a piezoelectric scanner. The motions consist of a slower horizontal scanning motion in the $x y$ plane and a faster vertical tracking motion in the $z$-axis. The bandwidth of the instrument is reduced due to the mass of the moving sample, especially in the vertical direction. In the stationary-sample-type AFM, the piezoelectric scanner carries the probe to scan, and the sample is completely fixed. Despite the fact that this kind of configuration provides advantages, false deflection should be prevented. The key point of the design is that the signal transduced from the PSD should only depend on the cantilever's deflection and not on the scanning motion. If the scanning motion may influence this signal, this undesirable side effect is then called false deflection or tracking error.

There are two major difficulties in the instrumentation of the stationary-sample-type AFM: The first one is to let the focused laser spot track an invariant point on the probe's cantilever, which is 3-D moving during operation. While the sample is far away from the probe and induces no deflection, how to let the laser beam, which is reflected from the moving cantilever, hit an invariant point (e.g., the center) of the PSD is the second difficulty. The goal of this paper is to develop a novel stationarysample-type AFM that satisfies these two conditions while limiting the false deflection.

\section{LASER BeAm Tracking System}

\section{A. Vertical Tracking}

The proposed beam tracking system is shown in Fig. 2. A piezoelectric oscillator is used to perform tapping-mode AFM. The probe is installed at the focus of the objective lens. The relative position of the probe and the objective lens is maintained by the aluminum-made lens holder, which is attached to the bottom of the $z$ scanner. The collimated beam, which is emitted by the LD and then adjusted by a four-axis laser mount, is kept parallel to the up-and-down motion of the $z$ scanner. The reflected beam from the probe is collimated by the same objective lens and then adjusted by mirrors 1,2 , and 3 


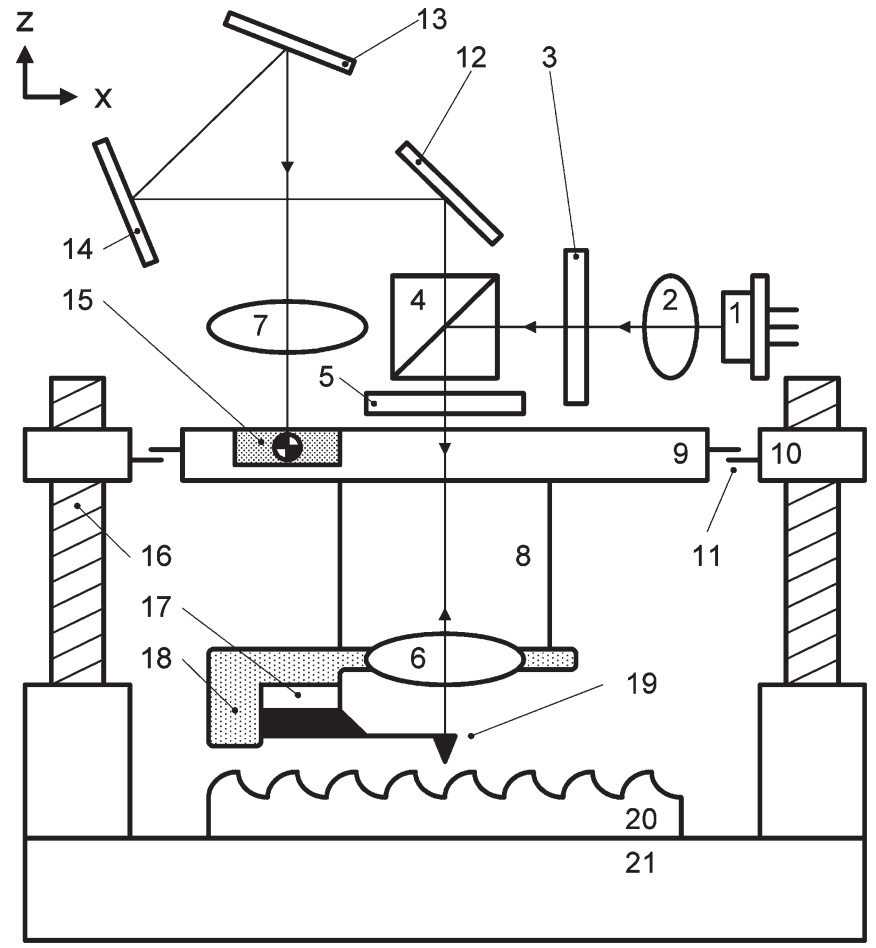

Fig. 2. Schematic diagram for the proposed system (1: LD; 2: collimation lens; 3: polarizer; 4: beam splitter; 5: $1 / 4 \lambda$ wave plate; 6: objective lens; 7: correction lens; 8: $z$ tube scanner; 9: moving part of the horizontal scanner; 10: fixed part of the horizontal scanner; 11: flexure guiding system; 12, 13, 14: mirrors 1-3; 15: four-segmented PSD; 16: approach mechanism; 17: piezooscillator; 18: lens holder; 19: probe; 20: sample; 21: vibration isolator).

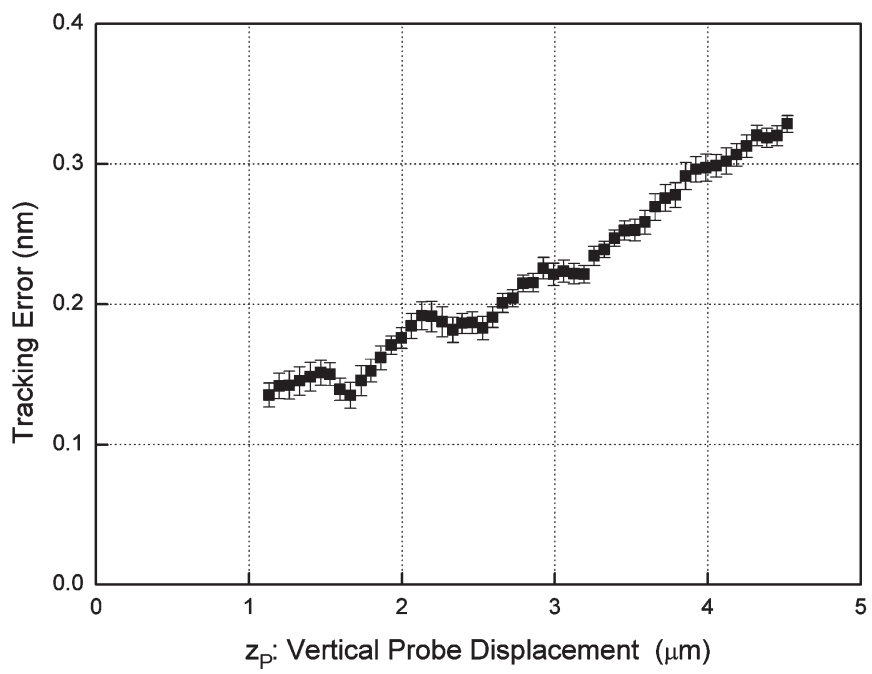

Fig. 3. False deflection in vertical void scanning.

to aim at the center of the PSD. The optical configuration will remain invariant during $z$ motion; hence, the tracking function is exactly achieved by the synchronized objective lens.

There should be no false deflection in vertical scanning theoretically. However, it is not eliminated because of the imperfection of optical elements. In order to measure false deflection, we operate void scanning, and the measured laser beam translation yields false deflection via (2). The experimental result of vertical false deflection is shown in Fig. 3. It is less than $0.3 \mathrm{~nm}$ over a $4-\mu \mathrm{m}$ scanning range. The testing procedure

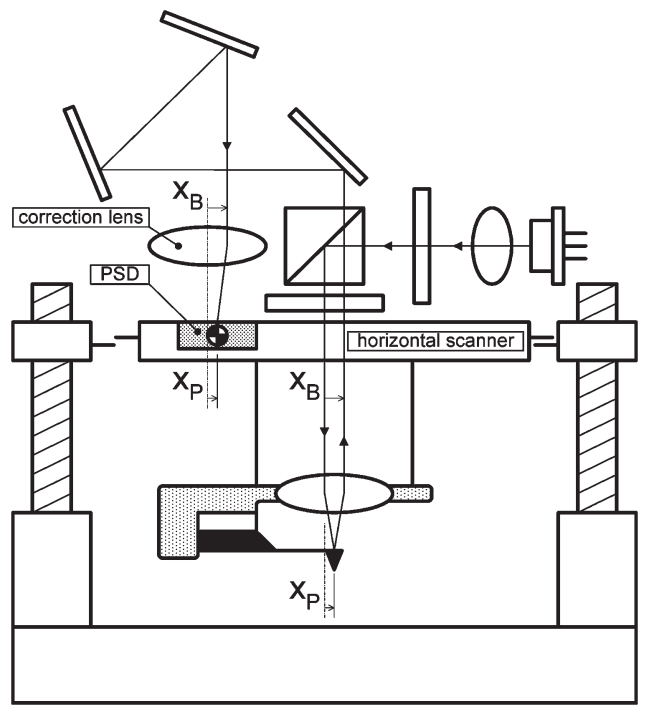

Fig. 4. Conceptual diagram during horizontal scanning. When the horizontal scanner drives the tip to travel by vector $x_{P}$, the reflected laser beam shifts $x_{B}$ correspondingly. Equation (8) describes that $x_{B}$ is a function of $x_{P}$. Since the PSD is attached to the horizontal scanner, it is also driven to travel $x_{P}$ synchronously. The correction lens is installed at a designed location in order to condense the laser beam from $x_{B}$ to $x_{P}$ so that the reflected laser beam can track the PSD while horizontal scanning.

is stated as follows: The probe is driven by the $z$ scanner following a sinusoidal function. The motion of the probe's tip can be described by second-order dynamics: $\ddot{z}_{t}+2 \zeta \omega_{n} \dot{z}_{t}+$ $\omega_{n}^{2} z_{t}=\omega_{n}^{2} z_{p}=\omega_{n}^{2}\left[Z_{p} \sin (\omega t)\right]$, where $Z_{p}$ and $\omega=62.8 \mathrm{rad} / \mathrm{s}$ are the amplitude and the frequency of the forcing function, respectively; $z_{p}$ stands for the displacement of the probe's base in the $z$-direction; and $z_{t}$ represents the $z$ displacement of the probe's tip. The numerical values of the damping ratio $\zeta=$ 0.0017 and the undamped natural frequency $\omega=82938 \mathrm{rad} / \mathrm{s}$ can be obtained from a system identification experiment, which is a built-in function of most AFM controllers. Substituting the values into the prior dynamic equation while $Z_{p}=2 \mu \mathrm{m}$, which represents the full swing of the $z$ scanner, the simulation result indicates that the maximum steady-state difference between $z_{t}$ and $z_{p}$ is only $10.4 \mathrm{pm}$ and can be omitted. Thus, the probe's tip and base move synchronously since the operation frequency is much lower than the resonant frequency. In other words, the inertia force is too small to bend the probe's cantilever in the dc region. During the test of Fig. 3, the sample is kept far away from the probe so that there should be no interaction force to induce deflection. Any measured signal is false deflection. A sensitive lock-in amplifier (SR830, Stanford Research System) is applied to detect the small signal below nanometer level.

\section{B. Horizontal Tracking}

During $x$ or $y$ scanning, the conceptual diagram of the optical configuration is shown in Fig. 4. When the horizontal scanner drives the tip to travel by a vector $x_{P}$, the incident beam and the reflected beam do not remain coaxial. The distance between their centers is called beam shift, which is denoted as $x_{B}$ and is roughly twice the vector $x_{P}$. Referring to Fig. 5, which illustrates the exact optical configuration around the probe, $\mathbf{R}$ 


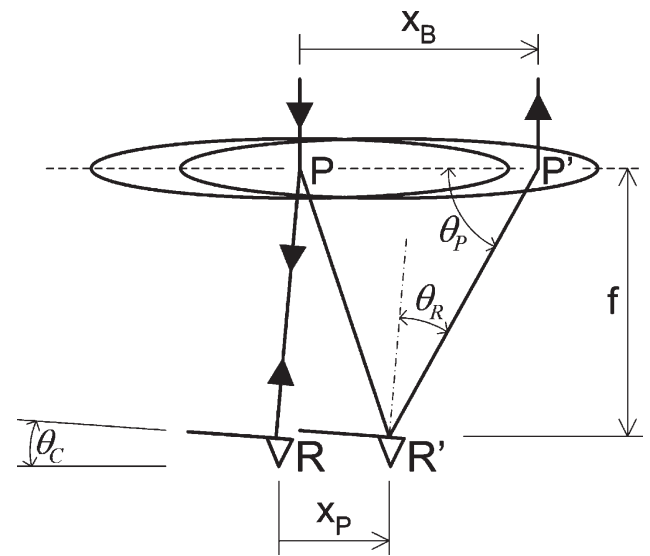

Fig. 5. Exact optical configuration during horizontal scanning.

denotes the tip position when the incident and reflected beam can be coaxial. Correspondingly, the reflected beam moves from $\mathbf{P}$ to $\mathbf{P}^{\prime}\left(x_{B}\right)$ when the tip moves from $\mathbf{R}$ to $\mathbf{R}^{\prime}\left(x_{P}\right)$. By applying some trigonometry to the triangle $\Delta \mathbf{P} \mathbf{P}^{\prime} \mathbf{R}^{\prime}$, we have

$$
\frac{\overline{P P^{\prime}}}{\sin \left(2 \theta_{R}\right)}=\frac{\overline{P R^{\prime}}}{\sin \left(\theta_{P}\right)}
$$

According to the optical configuration in Fig. 5, we obtain

$$
\begin{aligned}
\overline{P P^{\prime}} & =x_{B} \\
\overline{P R^{\prime}} & =\sqrt{f^{2}+\left(x_{P}-f \tan \theta_{C}\right)^{2}} \\
\theta_{R} & =\theta_{C}+\tan ^{-1}\left(\frac{x_{P}}{f}-\tan \theta_{C}\right) \\
\theta_{P} & =\frac{\pi}{2}-2 \theta_{C}-\tan ^{-1}\left(\frac{x_{P}}{f}-\tan \theta_{C}\right) .
\end{aligned}
$$

Substituting (4)-(7) into (3), $x_{B}$ is a function of $x_{P}$ as follows:

$$
\begin{aligned}
x_{B}\left(x_{P}\right)=\sqrt{f^{2}+\left(x_{P}-f \tan \theta_{C}\right)^{2}} & \\
& \cdot \frac{\sin \left(2 \theta_{C}+2 \tan ^{-1}\left(\frac{x_{P}}{f}-\tan \theta_{C}\right)\right)}{\sin \left(\frac{\pi}{2}-2 \theta_{C}-\tan ^{-1}\left(\frac{x_{P}}{f}-\tan \theta_{C}\right)\right)} .
\end{aligned}
$$

Referring to Fig. 4, the number of the mirrors should be odd to make $x_{B}$ and $x_{P}$ have the same direction; hence, (8) will hold. Three mirrors are chosen for this paper.

Referring to Fig. 4, we designed a correction lens to be installed at an appropriate location in order to condense the laser beam from $x_{B}$ to $x_{P}$ so that the reflected beam can track the PSD while horizontal scanning. Since the relation between $x_{B}$ and $x_{P}$ is approximately linear, as described in (8) and plotted in Fig. 6, the linear correction lens can function well. However, it is not perfect because the correction lens condenses the laser beam from $x_{B}$ to $x_{P}^{\prime}$ rather than to the exact $x_{P}$. The residue error between $x_{P}^{\prime}$ and $x_{P}$ induces the theoretical false deflection. The behavior of the correction lens can be expressed as $x_{B}=C \cdot x_{P}^{\prime}$, where $C$ is a constant that depends on the distance between the correction lens and the PSD. Theoretically, minimized false deflection can be obtained by installing the

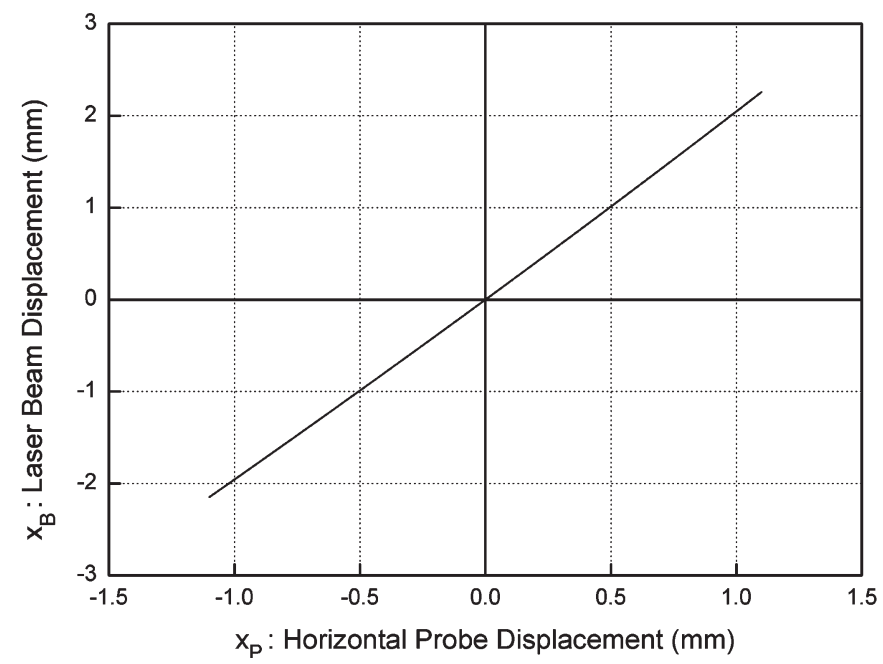

Fig. 6. Plot of (8). Referring to Fig. 4, a correction lens is used to condense the laser beam from $x_{B}$ to $x_{P}$ in order to cancel the false deflection. Since the relation of these two variables is approximately linear, the linear correction lens can function well. The residue error between the linearized equation $x_{B}=C$. $x_{P}^{\prime}$ and (8) induces the theoretical false deflection, which is plotted in Fig. 7.

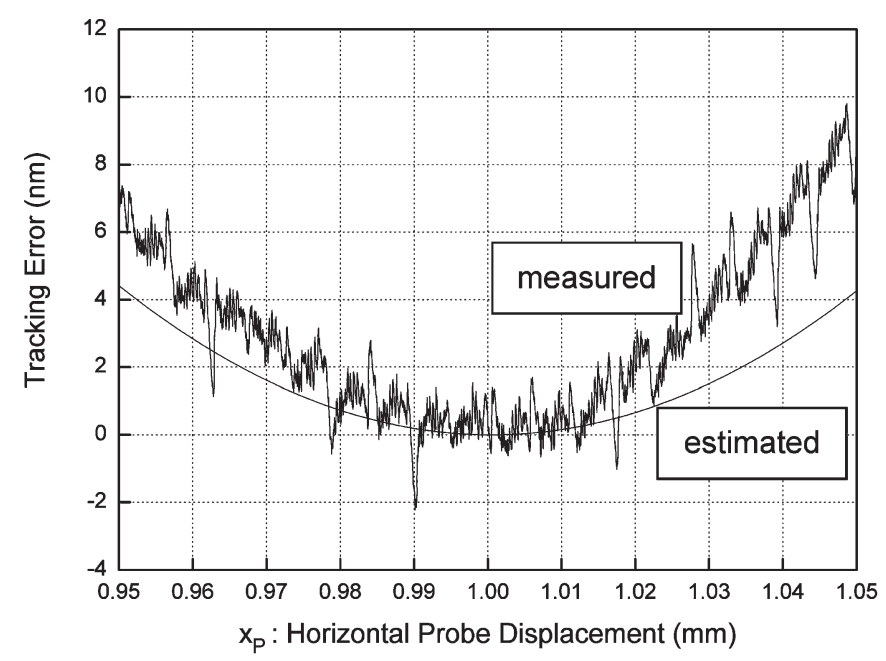

Fig. 7. False deflection in horizontal void scanning. The measured data has the same trend with the theoretical false deflection, which is depicted by the smooth line.

correction lens at a distance of $f_{C} / C$ above the PSD, where $f_{C}$ is the focal length of the correction lens. The shorter the $f_{C}$, the sharper the condensing angle of the correction lens, which implies that a longer $f_{C}$ may make the adjustment easier. Generally speaking, the larger diameter and thinner central thickness of this lens make it close to an ideal lens. However, the size of this correction lens cannot be increased unlimitedly, or mechanical interference may occur. A double-convex lens (DCX series, Edmund Company), which is especially designed for collimation, is thus chosen for this paper. Its effective focal length is $9 \mathrm{~mm}$.

Conservatively, 1-mm mechanical misassembly is assumed, and the horizontal scanning range is set to be $100 \mu \mathrm{m}$ (i.e., $x_{P}$ travels from 0.95 to $1.05 \mathrm{~mm}$ ). The estimated and the measured horizontal false deflections that result from the current setup are shown in Fig. 7. The measured false deflection is $12 \mathrm{~nm}$ over 


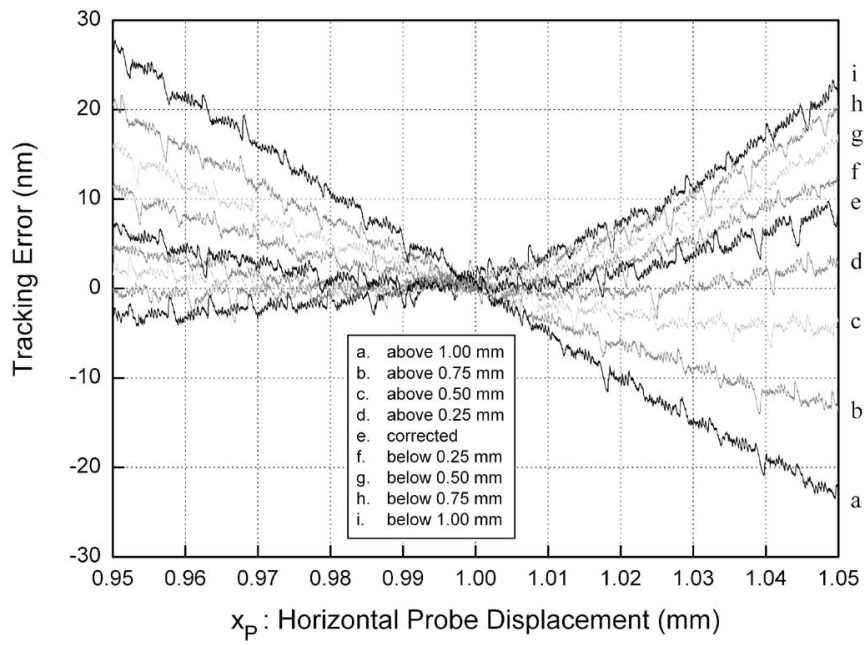

Fig. 8. False deflection that is induced by misinstallation of the correction lens. Line (e) represents the minimized false deflection when the correcting lens is installed at the appropriate location. Line (d) represents the false deflection when the correcting lens is installed $0.25 \mathrm{~mm}$ higher than the correct location. The other lines represent the case when the correcting lens is installed at other locations.

the 100- $\mu \mathrm{m}$ scanning range. Aside from the imperfection of the optical elements and mechanical misassembly, the out-of-plane motion of the $x y$ scanner can be a reason. In the test described in the next section, we inspect the sample in an $11 \times 11 \mu \mathrm{m}$ region. In this small region, the false deflection is comparable to the background noise and hence is negligible. It is mentioned that the correction lens should be installed at a particular location. The location of the correction lens is fine-tuned by a translation stage having a resolution of $10 \mu \mathrm{m}$. Misinstallation will induce a larger amount of false deflection in horizontal void scanning. Referring to Fig. 8, line (a), among the others, will be first obtained if we tune the correction lens downward, and the tracking error of line (a) is $50 \mathrm{~nm}$. If we continue to tune down the correction lens for $0.25 \mathrm{~mm}$, line (b) with a $35-\mathrm{nm}$ tracking error will start to appear. Continuing this procedure, we can collect lines (c), (d), ..., (i). The tracking error decreases as we tune down the correction lens but increases again if the correction lens is placed too low like line (i). After iterative trial and error, the minimized tracking error can be achieved, i.e., line (e).

\section{EXPERIMENTAL RESULTS}

Fig. 9 shows the experimental setup. Aside from the proposed stationary-sample system, we place an additional independent scanner (secondary scanner) under the sample. We use the same piezoelectric tubes $(4 \mu \mathrm{m} / 400 \mathrm{~V})$ as $z$ portions of both the primary and secondary scanners. If we turn on the primary scanner but turn off the secondary scanner, the proposed stationary-sample-type AFM is performed. If the scanners are switched in the opposite way, the hardware functions as the conventional scanning-sample system so that we can compare the performances of these two systems.

A commercial AFM controller (NanoScope IIIa, Digital Instrument) provides a signal access module, which is used to drive our hardware. The specimen is a standard grating with

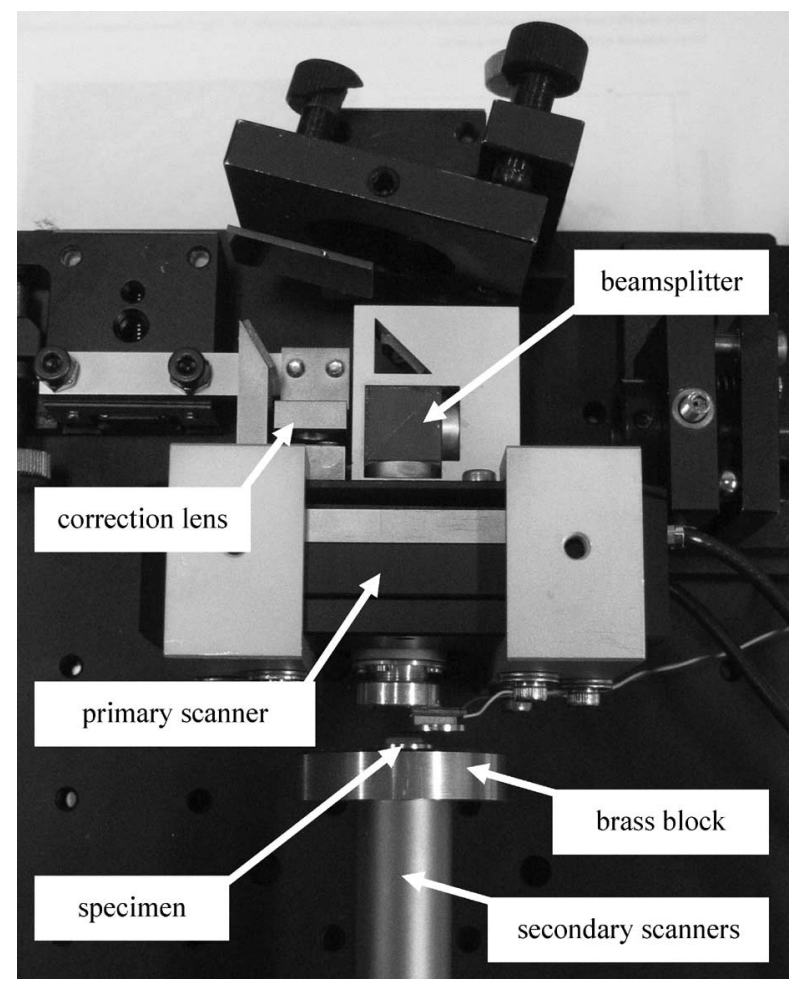

Fig. 9. Photograph of the experimental setup. The location of the correction lens is fine-tuned by a precision translation stage in order to minimize the false deflection. The brass block is used to simulate a heavy sample.

a step height of $26 \pm 1 \mathrm{~nm}$ and the horizontal pitch length of $3 \pm 0.1 \mu \mathrm{m}$. This specimen is attached on a $51-\mathrm{g}$ brass block, which is used to simulate a heavy sample. The vertical working frequency of the secondary scanner can be expected to decrease due to the greater mass it carries. In comparison, the mass carried by the primary $z$ scanner is only $3 \mathrm{~g}$, including the probe, the objective lens, and the lens holder. The following experimental results demonstrate the performance of our method: Two images [Fig. 10(a) and (b)] taken with different scanning speeds clearly indicate that the secondary $z$ scanner cannot follow the fast change of the surface topograph. In contrast, referring to Fig. 10(c) and (d), the proposed stationarysample-type AFM system performs well without profile distortion, even at high scanning speed.

\section{CONCLUSion}

We developed a novel laser beam tracking system for AFM to measure the deflection of the microprobe while moving in 3-D space. Using this methodology, the sample is fully stationary during operation. This characteristic implies that the instrument bandwidth will not be reduced by the mass of the sample; therefore, heavy samples such as 12 -in wafers can be observed directly by our system. In the past, the sample had to be cut into small pieces since it was too heavy, before it could be loaded into the moving-sample-type AFM. However, the cutting procedure may incur cracks in the divided samples and some pollution on the sample surface.

Relative to the existing literature, our system can demonstrate the highest scanning speed at $50 \mu \mathrm{m} / \mathrm{s}$ but with no 

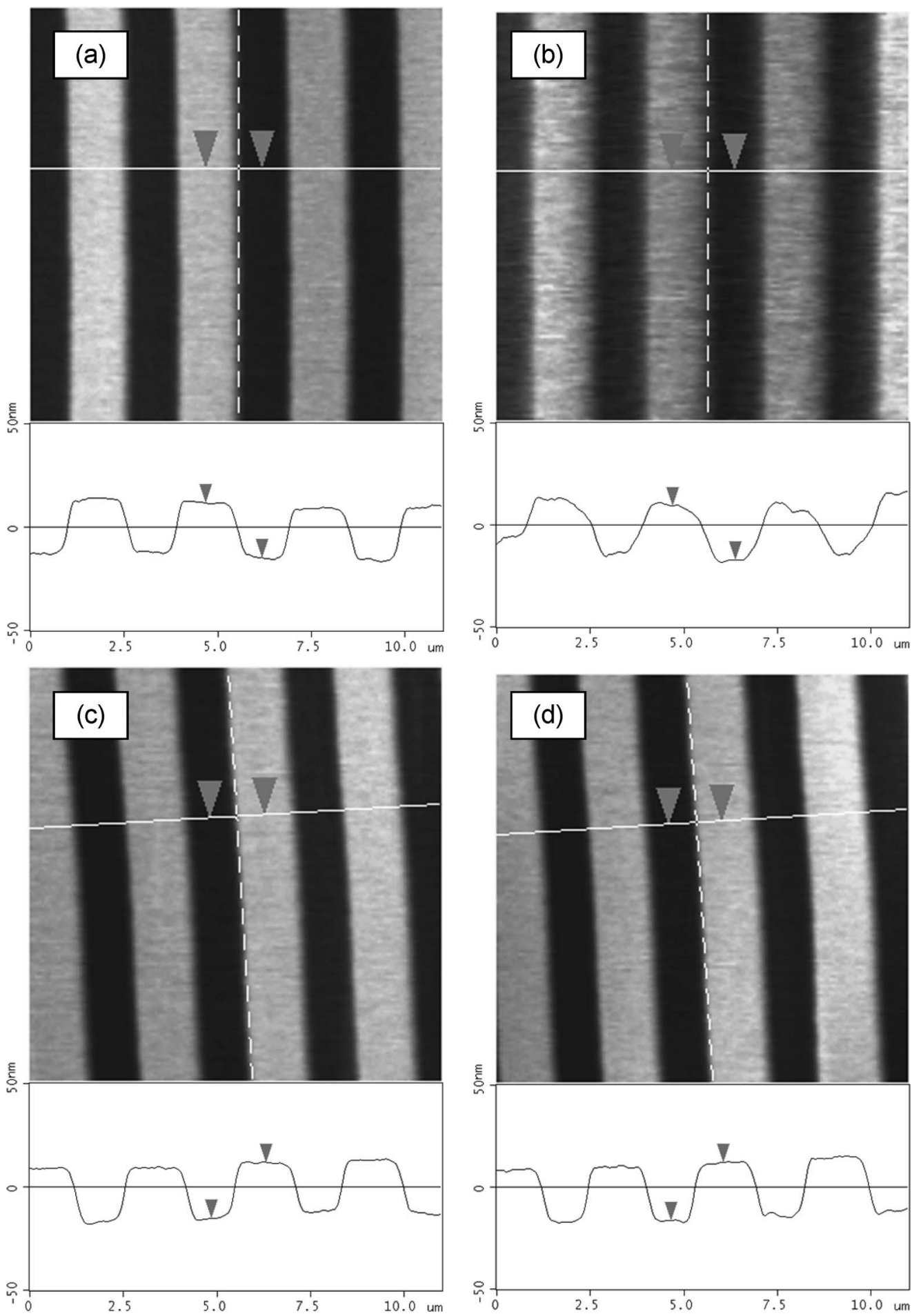

Fig. 10. AFM topographs and height profiles of a standard grating specimen at different scanning speeds and methods. Our method can track the trenches well at the speed of $50 \mu \mathrm{m} / \mathrm{s}$, while the conventional method cannot. (a) Conventional method at $5 \mu \mathrm{m} / \mathrm{s}$ in a horizontal direction. (b) Conventional method at $50 \mu \mathrm{m} / \mathrm{s}$ in a horizontal direction. (c) Proposed method at $5 \mu \mathrm{m} / \mathrm{s}$ in a horizontal direction. (d) Proposed method at $50 \mu \mathrm{m} / \mathrm{s}$ in a horizontal direction.

sacrifice in tracking accuracy. The vertical and horizontal false deflections are limited below 0.3 and $12 \mathrm{~nm}$ over the full scanning ranges of up to 4 and $100 \mu \mathrm{m}$, respectively. In addition, since our system adopts a flexure scanner, we can perform better in-plane motion than other systems, which can only use tubular piezoelectric scanners.

\section{ACKNOWLEDGMENT}

The authors would like to thank the reviewers for providing precious guidance, Dr. I.-S. Hwang and Dr. C.-S. Chang of Taiwan Academia Sinica for their advice on SPM fundamentals, and Taiwan Precision Instrument Inc. for providing the SPM controller to establish this research. 


\section{REFERENCES}

[1] G. Binnig, C. F. Quate, and C. Gerber, "Atomic force microscope," Phys. Rev. Lett., vol. 56, no. 9, pp. 930-933, Mar. 1986.

[2] P. S. Jung and D. R. Yaniv, "Novel stationary-sample atomic force microscope with beam-tracking lens," Electron. Lett., vol. 29, no. 3, pp. 264-266, Feb. 1993.

[3] J. Kwon et al., "Atomic force microscope with improved scan accuracy, scan speed, and optical vision," Rev. Sci. Instrum., vol. 74, no. 10, pp. 4378-4383, Oct. 2003.

[4] P. K. Hansma and B. Drake, "A new, optical-lever based atomic force microscope," J. Appl. Phys., vol. 76, no. 2, pp. 796-799, Jul. 1994.

[5] K. Nakano, "Three-dimensional beam tracking for optical lever detection in atomic force microscopy," Rev. Sci. Instrum., vol. 71, no. 1, pp. 137-141, Jan. 2000.

[6] T. Wang, S. Zheng, and Z. Yang, "A high precision displacement sensor using a low-finesse fiber-optic Fabry-Pérot interferometer," Sens. Actuators A, Phys., vol. 69, no. 2, pp. 134-138, 1998.

[7] T. Yoshino, K. Kurosawa, K. Itoh, and T. Ose, "Fiber-optic Fabry-Pérot interferometer and its sensor applications," IEEE Trans. Microw. Theory Tech., vol. MTT-30, no. 10, pp. 1612-1621, Oct. 1982.

[8] Digital Instruments, Fine Calibration Procedures for the SPM-A User's Guide, pp. 1-31, 1996. Support Note No. 217.

[9] H.-J. Butt and M. Jaschke, "Calculation of thermal noise in atomic force microscopy," Nanotechnol., vol. 6, no. 1, pp. 1-7, Jan. 1995.

[10] R. Lévy and M. Maaloum, "Measuring the spring constant of atomic force microscope cantilevers: Thermal fluctuations and other methods," Nanotechnol., vol. 13, no. 1, pp. 33-37, Feb. 2002.

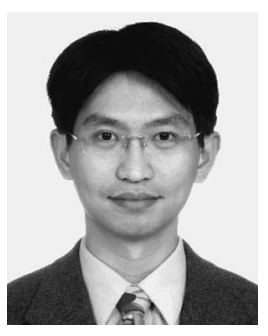

Shao-Kang Hung (S'03) was born in Keelung, Taiwan, R.O.C., in 1976. He received the B.S. and the M.S. degrees from the Department of Mechanical Engineering, National Taiwan University, Taipei, Taiwan, in 1998 and 2000, respectively. He is currently working toward the Ph.D. degree in the Department of Electrical Engineering, National Taiwan University.

Since 2000, he has been with Nanoscience Laboratory, Institute of Physics, Academia Sinica, Taipei, where he has been involved in the instrumentation of scanning probe microscopy. His research interests include optomechatronic systems, precision measurement, and control theory and applications.

Mr. Hung was a recipient of the Best Project Award of the National Student Hands-on Competition in 2003.

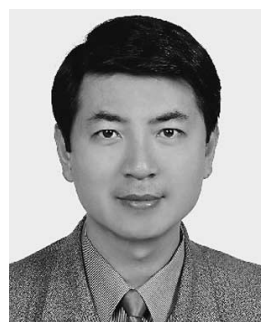

Li-Chen Fu (S'85-M'88-SM'02-F'04) was born in Taipei, Taiwan, R.O.C., in 1959. He received the B.S. degree from National Taiwan University, Taipei, in 1981 and the M.S. and Ph.D. degrees from the University of California, Berkeley, in 1985 and 1987, respectively.

Since 1987, he has been a member of the faculty of National Taiwan University, where he is currently a Professor in both the Department of Electrical Engineering and the Department of Computer Science and Information Engineering. He is also currently the Deputy Director of Tjing Ling Industrial Research Institute, National Taiwan University. His research interests include adaptive control, nonlinear control, precision measurement, visual tracking, control of robots, and flexible manufacturing system (FMS) scheduling.

Dr. Fu was a recipient of the Excellent Research Award from 1990 to 1993; the Outstanding Research Award from the National Science Council, Taiwan, in 1995, 1998, and 2000; the Outstanding Youth Medal in 1991, the Outstanding Engineering Professor Award in 1995, the Best Teaching Award in 1994 from the Ministry of Education, the Ten Outstanding Young Persons Award of Taiwan in 1999; the Outstanding Control Engineering Award from the Chinese Automatic Control Society in 2000, and the Lee Kuo-Ding Medal from the Chinese Institute of Information and Computing Machinery in 2000. During 1996-1998 and 2000, he was appointed AdCom member of the IEEE Robotics and Automation Society. He served as the Program Chair of the 2003 IEEE International Conference on Robotics and Automation and the Program Chair of the 2004 IEEE Conference on Control Applications. He has been the Editor of the Journal of Control and Systems Technology and an Associate Editor of the prestigious control journal Automatica. Since 1999, he has been an Editorin-Chief of a new control journal, called the Asian Journal of Control. 